\title{
Piloting different approaches to personalised offender management in the English criminal justice system
}

Chris Fox, Professor of Evaluation and Policy Analysis, Manchester Metropolitan University

Jordan Harrison, Research Assistant, Interserve Justice

Caroline Marsh, Independent Consultant

Andrew Smith, Research Associate, Manchester Metropolitan University

Corresponding author: Chris Fox

c.fox@mmu.ac.uk

Manchester Metropolitan University, PERU, Geoffrey Manton Building, 4 Rosamond Street West, Manchester, M15 6LL, UK

\section{Abstract (200 words max)}

Various approaches to personalisation are well-established in the UK social care sector and are now starting to 'travel' to othe sectors. In this paper we report findings from an evaluation of a pilot to test elements of personalisation in the management of offenders in probation services within the English criminal justice system. Following a review of evidence from social care, three different approaches to personalised-practice were developed and tested on a small-scale in three separate sites. The evaluation finds that all three approaches were implemented reasonably successfully, but challenges were identified including that personalised approaches are more time-consuming, that staff need support to exercise professional discretion and that balancing greater choice with managing criminogenic risk requires new ways of conceptualising the relationship between case manager and service user. Overall, 'deeper' approaches to personalisation such coproduction will take time to emerge. This paper makes two important contributions to the debate on personalisation in public services. First, it addresses the question of how transferable the concept of personalisation is from the social care sector to other sectors in the UK, in this case the criminal justice system. Secondly, it outlines a methodology for developing and evaluating personalisation pilots, prior to wider rollout.

\section{Key words}

Personalisation, offender management, desistance, probation, evaluation

\section{$\underline{\text { Introduction }}$}

In this paper we report findings from an evaluation of a pilot to test elements of personalisation in the management of offenders in probation services within the English criminal justice system.

Personalisation can mean many things. Most simply, personalisation means that public services respond to the needs of clients, rather than offering a standardised service, and can be seen as a response to the end of the age of deference, increasing customisation available in consumer goods, and the idea that by designing services for the average, they end up fitting no-one (Prime Minister's Strategy Unit 2007, Rose 2016). Pearson, 
Ridley and Hunter (2014) identify three levels of personalisation: prevention, participation and choice. First, personalisation can be seen as a means of prevention, designed to build an individual's capacity to manage their own lives. Secondly, 'co-production' is a means of allowing people with complex needs to participate in shaping and delivering their service solutions. This involves "the reciprocal relationships that build trust, peer support and social activism with communities" (Pearson et al. 2014 p.18). Thirdly, personalisation embeds consumer choice linked to a broader discourse that emphasizes the potential of personalisation to transform relationships between the state, service providers and service users.

In the UK, personalisation as a trend in public service reform has been most obvious in adult social care. A widely known aspect of personalisation in social care is the attempt to give people greater choice and more control over services through direct control of the money allocated to their support through a personal budget based on individual care needs. Pearson et al. (2014 p.42), note that, in social care:

Offering people an individual budgetary allocation and giving them the opportunity to say how this should be spent to meet their care needs may seem simple but is a radical departure from traditional service culture.

The National Audit Office (2016) reports that there were about 500,000 adults whose social care services were paid for through local authority personal budgets in 2014-5 and the political case for advancing personalisation in adult social care in England has been largely won (Glasby et al., 2009).

However, the model has been criticised. Some are concerned at what they see as increased emphasis on individuals as consumers and reduced collective ethos (Roulstone and Morgan 2009, Slay 2011). Implementation of personal budgets without other key changes has been shown to result in limited positive change Fox (2012). Fox (2012) has emphasized that personalisation in the UK social care sector, tends to focus on personal budgets and gives insufficient attention to relationships, community life and responsibilities.

Turning to commissioning, local government authorities struggle to manage and support the local care market and this has led to less choice in some areas (National Audit Office 2016). There is growing realization in the UK social care sector that personal budgets are most effective in reshaping provision when coupled with commissioning activity which helps providers to better understand how to provide more personalised services. This can include efforts to promote and support the development of start-ups and micro-enterprises (Fox, Fox and Marsh 2013). It is acknowledged that the evidence base to support better outcomes through personalisation in social care is limited and based on personal narratives. Another strand of criticism concerns the limited evidence-base for personalisation. The National Audit Office (2016) found that while user-level data indicate that personal budgets benefit most users, when these data are aggregated, there is no association between higher proportions of users on personal budgets and overall user satisfaction or other outcomes.

Despite these various criticisms the narrative of personalisation has since travelled across a range of policy areas including welfare-to-work programmes and homelessness where, it is noticeable that a number of Payment by Results programmes and Social Impact Bonds have incorporated the concept of personalisation (see for instance, Department for Communities and Local Government 2014). Across these areas, personalisation encompasses a range of new ways of designing services, which can provide both what Leadbeater (2004) describes as 'shallow' approaches such as providing people with a more customer-friendly interface and giving users more say in navigating their way through services, through to 'deep' approaches that give users more direct say over how money is spent or position service users as co-producers of a service.

\section{About this paper}

This paper starts by describing the pilot that has been evaluated. It then continues with a short discussion of key findings from a review of theory and practice in the social care sector. We then set out the evaluation 
methodology before moving on to describe key findings and analysis. We finish with a discussion and conclusions.

This paper makes two important contributions to the debate on personalisation in public services. First, it addresses the question of how transferable the concept of personalisation is from the social care sector to other sectors in the UK, in this case the criminal justice system. Secondly, it outlines a methodology for developing and evaluating personalisation pilots, prior to a wider roll-out.

\section{The personalisation pilot}

This pilot is taking place in the English criminal justice system (from 2016 to 2020) in a number of newly formed Community Rehabilitation Companies (CRCs) created as part of the UK government's 'Transforming Rehabilitation' strategy (Ministry of Justice 2013). Interserve has responsibility for five of the new CRCs. CRCs are responsible for managing low and medium risk offenders subject to community sentences or offenders who are released from prison into the community under licensing conditions. Since taking over management of CRCs, Interserve has developed a service delivery model, Interchange, which promotes asset-based and more personalised approaches to working with service users. Alongside this Interserve has committed to also developing and piloting a more radical personalised approach to working with service users. To pursue this aim the research team have worked with Interserve to create a three-phase approach to developing and evaluating a model for a more radical approach to personalised working with offenders. This paper discusses findings from the first phase. In the second phase of the project, learning from the five conceptual models will inform a single, larger scale pilot of a fully-formed model of personalised working that will be implemented during 2018. This larger-scale pilot will be implemented and evaluated in one site, but with sufficient staff and service users to design an impact evaluation. In the third phase a larger roll-out of the pilot (where the evaluation team has less control of the implementation process) will test the sustainability of the model at scale.

In the first phase $(2016$ - 2017) we reviewed theory and practice in the social care sector and used this to inform the development of five personalisation pilots, each of which was designed to test an element of a personalised approach to working with offenders. These five elements were each implemented and evaluated in a separate site, each in a different Community Rehabilitation Company. Implementation was on a small scale with a handful of staff and service users involved in implementing each element. The aim of these pilots was to test the viability of each personalisation element in a 'real world' setting, to established whether the elements were implemented successfully and to learn implementation lessons. The first three elements we tested were as follows:

1. Person-centred Practice: Person-centred practices were adopted by selected and trained staff and managers within a single team managing a mixed caseload. A strong emphasis was placed on staff and service users co-producing a rehabilitation plan and professional discretion to tailor supervision to the holistic needs of the service user. The pilot explored the effect of person-centred practice on the process of co-production of rehabilitation plans for service users as well as on staff in the CRC.

2. Person-centred Practice with access to an Enabling Fund: In addition to the model of personcentred practice implemented in the first pilot, this pilot also included an enabling fund, a form of personal budget. The enabling fund was designed to support rehabilitative goals that could not be progressed through existing services such as accredited or non-accredited programmes, welfare payments or referrals to other agencies. The pilot explored the effect of person-centred practice and access to a form of personal budget on the process of co-production for staff and service users. 


\section{Person-centred Practice and an enabling fund for women delivered by a third party Women's}

Centre: In this pilot we examined the implementation of a person-centred approach, supported by access to a form of personal budget and its effect on co-production for a service user group with distinct and complex needs and whether delivery by a third party leads to distinct processes of coproduction. As with the second pilot, the third pilot concept introduced person-centred practice although in this case focussing solely on a cohort of women service users with the support of an enabling fund. In this instance, the enabling fund was controlled and administered by a third-party women's centre charity. In this pilot, person-centred practice was adopted by selected staff and managers within both the women's centre and the CRC.

The first three elements that we piloted concentrated on the operationalization of personalised practice in probation practice and are the focus of this paper. The other two conceptual models we tested in the first phase concentrated on the supply-side of the 'market' for personalised services and findings from these two pilots will be reported in due course.

\section{Learning from the social care sector}

Prior to designing and evaluating the personalisation pilots we undertook a review of evidence and theory of personalisation from the social care sector. A detailed set of findings can be found in Fox and Marsh (2016).

Fox, Fox and Marsh (2013) have argued that the criminal justice sector could learn from social care when considering the challenge of reforming the criminal justice system and developing innovative approaches to offender rehabilitation, although this is not without challenges (Fox and Grimm 2015). The social care sector used to rely heavily on institutional settings; the criminal justice sector still does - the UK prison population in August 2017 remained close to an-all time high at 86,237 (Ministry of Justice 2017). As Fox et al. (2013), note, before 'self-directed support' became the dominant narrative within the social care sector, a 'one size fits all' model of centrally planned and organized services prevailed, with little individual or family control. Social care was characterised by a medical model of disability and low expectations of people with long term health conditions, disability or ageing.

We see comparison between social care before self-directed support and the criminal justice system now in the standardisation of interventions and attitudes to service users, particularly in relation to whether service users are seen in terms of their deficits or assets.

In the current English Criminal Justice System the Risk, Need and Responsivity (RNR) principles (Andrews and Bonta 2006) are highly influential. The 'risk principle' assumes that higher risk offenders have a broader range of problems and these tend to be deeper rooted so they should receive a higher and more intense 'dose' of treatment than lower risk offenders. Risk factors are viewed as discrete, quantifiable characteristics of individuals and their environments that can be identified and measured (Ward and Maruna 2007). The 'need principle' assumes that treatment has larger effects if it addresses the criminogenic needs of the offender. Responsivity is concerned with how an individual interacts with the treatment environment - effective treatment can bring about change in the targeted criminogenic needs when it is responsive to the learning styles and characteristics of the offenders treated. They have a strong focus on managing criminogenic risks and, it has been argued, are based on a rather restricted and passive view of human nature (Ward and Maruna 2007).

The RNR model has led to increasing 'standardization' of rehabilitation interventions. It seems to contradict research and theory about why and how people desist from offending that suggests a more personalised 
approach to working with offenders is required. Maruna (2001) describes the importance of offenders' internal 'narratives' in supporting either continued offending or desistance. In his research with ex-offenders he found that individuals needed to establish an alternative, coherent and pro-social identity in order to justify and maintain their desistance from crime. McNeill $(2009$, p.28) asserts that in this context "One-size-fits-all processes and interventions will not work." However, a challenge posed by desistance research is that it is "not readily translated into straightforward prescriptions for practice" (McNeill and Weaver 2010 p.6). This is not necessarily problematic, because developing a prescriptive model of practice would undermine personalisation. In the first three elements of personalisation that we tested we concentrated on personalisation at the level of individual probation practice and assume that probation practice is informed by an understanding of desistance:

'The practitioner has to create a human relationship in which the individual offender is valued and respected and through which interventions can be properly tailored in line with particular life plans and their associated risk factors.' (McNeill 2009 p.27)

Therefore more personalised approaches are required where tailored life plans that recognize an offender's assets as well as their deficits (criminogenic risk factors) are central (McNeil 2009). Co-production is key to this process, although negotiating meaningful co-production in the criminal justice system presents many challenges (Weaver 2011). Drawing on the experience of the social care sector we also explore how different approaches to using a form of personal budget might support person-centred practice.

In social care personalisation reforms have taken 'strengths' based approaches that emphasize people's selfdetermination and strengths to move away from a focus only on what is lacking, personal shortcomings, 'deficits' and 'need'. There are obvious parallels between the literature on desistance from offending (e.g. Maruna 2001) and asset-based and personalised models of social care. These include: recognizing that rehabilitation is a process; focusing on positive human change and avoiding negative labelling; recognizing the importance of offender agency, recognizing the importance of offender relationships (professional and personal); and developing offenders' social capital.

Comparing reforms across the two sectors, we see further parallels. In both there have been attempts to diversify the provider-base using commissioning strategies such as the introduction of market testing. In both sectors there is debate about community-based services and the relationship between service users and their communities (Fox and Marsh 2013).

As Fox and Grimm (2015) note, at first glance the criminal justice system might not seem a promising sector for social innovation. The requirements of justice evoke concepts such as certainty, control, consistency and adherence to well-defined processes that are not necessarily compatible with innovation. Nevertheless there is a rich tradition of social innovation in the criminal justice system that embodies many key dimensions of 'social' innovations, such as the Restorative Justice movement (Mulgan, Tucker, Ali and Sanders 2007). Circles of Support, Community Justice and Justice Reinvestment, particularly in its early forms as advocated by Tucker and Cadora (2003), might also be characterized as forms of social innovation.

Key to social innovation are new processes that make use of social relations. In relation to re-offending, the desistance literature has emphasized the importance of human relationships both between workers and offenders and between offenders and those who matter to them (McNeill, Farrall, Lightowler, and Maruna 2012). Drawing on experience of increasing choice and control of care and support through the use of personal budgets from the social care sector, a new model is being developed for more personalised approaches to offender rehabilitation (Fox and Marsh 2016, Fox et al. 2013). Co-production will be key to this, although negotiating meaningful co-production in the criminal justice system presents many challenges (Weaver 2012). This requires new approaches to assessment and sentence planning, new training for staff and rethinking the language of practice (McNeil et al. 2012). 
It is not suggested that the social care model can be transposed directly to criminal justice. Compared to social care, the criminal justice system presents additional challenges in terms of the need to manage risk of harm to protect the public and to punish as well as reform through delivering the sentence of the court, and where interactions between clients and agencies are mandated. Rather a new model needs to be developed. Fox and Marsh (2016) argue that key considerations include: developing a culture of person-centred support; increasing access to community based services to increase social inclusion; developing appropriate choice and flexibility about how interventions are delivered; ensuring that a wide range of interventions is available; and providing access to enabling resources based on individual needs for support, whether this is through a personal budget or other means.

\section{Methodology}

Too often, pilots in the criminal justice system have been implemented prematurely with insufficient time and resource put into first developing a sound theory of change and then testing key elements prior to a larger pilot. We have therefore followed an adapted version of the model of piloting set out by the Education Endowment Foundation (EEF) in its guidance (EEF 2015). This specifies three types of trial conducted in sequence in the development and testing of new interventions. These are pilot, efficacy and effectiveness trials.

The findings set out in this paper are from the pilot trial, which is an early-stage study conducted in a small number of settings with the objective to "develop and refine the approach and test [an intervention's] feasibility" (EEF 2015 p.3). Qualitative research is expected to be the predominant data collection methodology. The idea is to test whether a new intervention has potential, where this has yet to be ascertained. In our scenario tesedt a number of 'personalisation' elements (described above). Each concept was piloted with a small number of cases (defined variously as service users, Case Managers or probation teams) selected purposively to allow testing of key theoretical constructs. Emphasis was placed on implementation evaluation, including the issue of implementing models of personalisation in a criminal justice setting where culture change will be key.

Evaluation of each personalisation concept began with a 'theory of change' (Weiss 1995) exercise. The theory of change approach draws on US work by Connell and Kubisch (1998) and Weiss (1995), among others and is becoming widely used in UK policy evaluation studies (eg Mackenzie and Blamey 2005). A theory of change for each personalisation pilot was developed following an initial review of relevant literature from social care and criminal justice management (described above) and a series of workshops involving practitioners and managers from CRCs who had been tasked with working with the research team to develop the interventions to be tested in each pilot. The evaluation team returned to the theories of change throughout the evaluation and various updates were made. The final versions are presented later in this report.

The main focus of the evaluation was on implementation evaluation that sought to describe the programme and whether or not it was delivered as intended to the targeted recipients (Scheirer 1994, cited in Rossi et al. 2004). Conducting an implementation evaluation allows the evaluator and stakeholders to measure whether an intervention has reached its target population, in this case service users, and to develop understanding of the organisational context in which the intervention may achieve its desired effect or bring about the greatest impact (Fox, Grimm and Caldeira 2016). This was done by looking to understand a wide range of complex factors including systems, processes and personal interactions situated within the context of the CRCs being tested, to increase understanding and inform the scope of the next phase of the pilot: an efficacy trial. To assess this complexity, the evaluation of the Personalisation PoC phase was undertaken using a dual methods strategy comprising: 
- 58 interviews with service users, staff and managers involved in implementing the personalisation concepts of which 30 were undertaken early in the pilot and 20 were follow-up interviews undertaken towards the end of the pilot. Interviews with staff and managers were done predominantly over the telephone whereas interviews with service users were mostly done face-to-face. Interviews were semi-structured. Interviews with staff and managers took between 30 minutes and an hour, interviews with service users tended to be shorter, around 25 minutes. Interviews were recorded and either detailed notes or transcripts were produced.

- $\quad$ There were 5 observations of supervision sessions between Case Managers and service users undertaken mid-way through the pilot. Observers were provided with a template highlighting key elements of the process and offering guidance on completing write-ups. The activity was then recorded in as much detail as possible.

- A total of 40 psychometric questionnaires were administered to staff and service users involved in implementing the personalisation concept of which 23 were undertaken early in the pilot and a further 17 completed at the follow up stage. Three different psychometric measures were used in the personalisation pilots. Psychometrics were used to generate data on 'hard to measure' concepts such as well-being (for service users), self-efficacy (for Case Managers and service users) and work engagement (Case Managers), all of which had been identified as potentially important during the Theories of Change work. Self efficacy was measured using the the General Self-Efficacy Scale (Schwarzer \& Jerusalem,1995), wellbeing using the Short Warwick-Edinburgh Mental Well-being Scale (University of Warwick, 2015) and work engagement using the Shortened Utrecht Work Engagement Scale - UWES-9 (Schaufeli \& Bakker, 2004). Individuals within each group (staff/volunteers and service users) were asked to complete a paper-based questionnaire that incorporated the relevant psychometric measures near the beginning of the intervention and prior to the end of the evaluation period. Typically the gap between questionnaires was 16 weeks. It was not expected that sufficient psychometric data would be collected for statistical inference to be made, or for empirical relationships between the different variables to be established. Rather the aim for this early pilot stage of the project was to evaluate the administration and use of the psychometrics within the context of the pilots, and to trial their use pending a larger scale data collection in the next phase of the research.

- $\quad$ Case file analysis was undertaken on all 27 service users involved in the pilot. This included data used to construct Offender Group Reconviction Scale (OGRS) scores, which includes data on previous offending history and current offence, and data from the Offender Assessment System (OASys), which captures a range of service user needs.

Prior to engagement, both staff and service users were presented with an information sheet documenting the purpose of the study, how data collected would be stored and used, their right to withdraw from the study and contact details should they require more information and/or encounter any other issues. All evaluation participants were asked to sign a consent form confirming they had read and understood the information being presented and that they consented to participate. Consent forms were signed in person, scanned and collated. Participants were informed of their right to withdraw at any given stage.

All qualitative data from interview transcripts and observation write-ups were imported into the computer software package Nvivo for analysis. A 'coding tree' was developed based on the evaluation research questions and theories of change exercise. Initial coding was undertaken and the coding structure was then reviewed and allowed to develop in a grounded manner to allow for emergent themes to be explored and refined. Data from the psychometric questionnaires were analysed in SPSS. Means and standard deviations were calculated and the results compared to previous studies. Data from case files was put into an Excel spreadsheet. The small number of cases meant that only simple, descriptive analysis was undertaken and tables of data are not reproduced in this report to preserve anonymity of service users. 
The evaluation was led, and much of the data collection and analysis undertaken, by the authors. In addition a group of staff from the Pilot Sites were recruited to participate in the evaluation work. Many of this group also had some involvement in the delivery of the pilot and the intention was to introduce an element of participatory action research (Smith et al. 2010) into the evaluation design. The probation staff received four half-day training sessions on evaluation led by one of the research team. The key strength of this evaluation team was that many were practitioners and managers involved in implementing the project. This gave them an additional level of insight into the workings of each pilot. A practical limitation was that staff were busy and and so scheduling time for evaluation work was often challenging. The team was also aware of the complexity of working as evaluators and implementers and issues of confidentiality and power dynamics were discussed during training.

\section{Developing the pilots}

Commencing from January 2017, three offices, one each in three Interserve led Community Rehabilitation Companies (CRCs) were selected to pilot elements of person-centred practice. The selection of the office in each CRC where the pilot would be run was pragmatic. At the time of the research, Community Rehabilitation Companies were newly established and had taken over staff and offices from previous Probation Trusts. Factors influencing site selection were that there was a reasonably settled team and that any office moves had been completed.

\section{Person-centred practice pilot}

In Pilot Site 1 person-centred practices were adopted by selected and trained staff and managers within a single team managing a mixed caseload. A strong emphasis was placed on staff and service users co-producing a rehabilitation plan and using professional discretion to tailor supervision to the holistic needs of the service user. The pilot explored the effect of person-centred practice on the process of co-production of rehabilitation plans for service users as well as on staff in the CRC.

To support this way of working a set of 'person-centred practice tools' were created by a trainer from the CRC, working with an external organisation with experience of developing personalised models of working in the health and social care sector. These tools had been used in social care setting, but were adapted for use in probation. Staff were encouraged to used a selection of the tools in their supervision sessions with service users to initiate a range of conversations. Included were tools to: help service users understand their significant relationships and plan how to build stronger ones; identify what makes a good or a bad day; identify assets; and, set personal goals. The use of these tools was not prescriptive; they were available to use depending on what staff and service users judged would best lead to a co-produced enabling plan to support rehabilitation. Supervision was expected to be delivered by means of face-to-face appointments where possible and contact to be in accordance with the service users order/licensing conditions. On the whole appointments were intended to be conducted in a one-to-one setting although in certain circumstances additional support staff such as drug and alcohol workers or other partner agency staff might attend.

The trainer and external organisation collaborated with Interserve staff to deliver training on person-centred practice. All staff involved in the pilot, including managers, attended an initial one day training session. Front line staff took part in two further in-depth training sessions delivered in a group setting. To allow for development and continuous improvement, practitioners were presented with a series of e-learning modules aimed at expanding their understanding of person-centred practice and encouraged to keep a reflective log to highlight any significant issues or challenges they faced. Once the pilot commenced further support was offered throughout the pilot by a small project management team who undertook regular site visits and instigated regular catch ups with front-line staff and their managers using a mix of telephone, email and skype. 
The Interserve internal trainer also observed Case Manager practice with service users on the pilot in two of the CRCs and provided developmental feedback to the Case Managers.

\section{Person-centred Practice with access to an enabling fund}

In Pilot Site 2 staff were provided with the same tools and training to support person-centred practice as described in Pilot Site 1 (above). In addition, this pilot also included an enabling fund. The enabling fund was designed to support rehabilitative goals that could not be progressed through existing services such as accredited or non-accredited programmes, welfare payments or referrals to other agencies. The pilot explored the effect of person-centred practice and access to a form of personal budget on the process of co-production for staff and service users.

Service users could either use the fund individually or pool it together with other service users. The total amount available per service user was determined in advance of the pilot, with the maximum amounts linked to the banding of the service user. The total amount available to service users was as follows; Band 2 - $£ 200$, Band $3-£ 600$ and band $4-£ 1200$. To access the funding, service users had to work in collaboration with their Case Manager (and key worker in Pilot Site 3) to identify areas of need and goods and/or services that met the requirements included in their enabling plan. Guidance and the details on the exact amount available per service user were distributed to Case Managers and key workers who were briefed by the project team on the purpose behind the enabling fund. All guidance, written and verbal, stressed the idea that staff and service users should collaborate on decisions about when and how to use the fund. The principles regarding the use of the enabling funds were simple - they were to be used on goods and services that were safe, legal, not available through other means (such as referral to community services delivered by another agency), and clearly linked to a goal or goals in the rehabilitation plan.

To increase learning and better understand the process by which money was spent, some service users in the Pilot Site 2 pilot who had access to the enabling fund were told of the amount available to them whereas others were not. The purpose of this was to avoid 'spending to the ceiling' and to identify whether having prior knowledge of the allocated budget influenced the way in which service users made use of their fund. The intention was that half of the service users would be informed about the amount of funding available and half would not. However, in reality just one service user was made aware of the amount allocated to them. This was largely due to practitioners failing to disclose information about the fund as expected, an issue discussed further later in this paper.

\section{Person-centred Practice and an enabling fund for women delivered by a third party Women's Centre}

In Pilot Site 3 staff were provided with the same tools and training to support person-centred practice as described in Pilot Site 1 (above). As in Pilot Site 2, Pilot Site 3 also introduced an enabling fund. In this instance, the enabling fund was controlled and administered by a third-party women's centre charity. Staff at the women's centre received the same tools and training on person-centred practice. This pilot examined the implementation of a person-centred approach, supported by access to a form of personal budget, and its effect on co-production for a service user group with distinct and complex needs and whether delivery by a third party leads to distinct processes of co-production.

\section{Staff and service users}

At each Pilot Site one Case Manager and one Senior Case Manager were responsible for managing the pilot caseload. At Pilot Site 3 a Key Worker from the womens' centre was also involved. All staff involved in 
delivering practice were female. The average time staff had been in their current role was 4.6 years (range 9 months to 14 years). The average time staff had worked in the sector was approximately 8 years (range 3.5 years to 17.5 years). An established psychometric measure to assess work engagement (UWES-9) was administered to staff and volunteers before and after their engagement in the personalisation project. Of the 8 who participated, 6 completed the questionnaire pre intervention and 7 post intervention. Mean scores for all responses (including matched and unmatched pairs) were 4.48 (S.D. 0.81) pre-intervention and 4.19 (S.D. 0.55) post-intervention. The numbers involved make it unwise to draw any conclusions, but the concept of using a psychometric as part of an evaluation of personalisation was successfully tested.

Each pilot sought to work with between 10 and 12 service users. Service users were eligible for the pilot if they were sentenced by the court to a community order that included a rehabilitation activity requirement and they were taken on to the pilot over a period of several weeks. All eligible cases allocated by the National Probation Service (NPS) to the pilot Case Managers in the CRC were brought on to the pilot until a sufficient number of 'starts' was achieved on each pilot. Once new cases were referred to the pilot local leads assigned them to either a Case Manager or Senior Case Manager dependant on the needs, complexity and risk posed by the service user. It was challenging to achieve target numbers of service users within project timescales. Pilot Site 1 and 2 , which had originally intended to only recruit males also recruited females to increase numbers.

The pilot reached a total number of 27 service users; nine in Pilot Site 1, 11 in Pilot Site 2 and 7 in Pilot Site 3. In total the pilots worked with 14 males and 13 females. These proportions are different to the general caseload across the 3 CRCs where approximately 86.4 percent of clients were male. The average (mean) age of service users was 32.6 which is very similar to the average age of the general caseload of service users across the three CRCs Over the course of the project six service users disengaged for various reasons including noncompliance and ill health. The offences that services users had been convicted of varied. Common offences included stealing from shops and stalls (shoplifting), common assault and possession of drugs. The Offender Group Reconviction Scale (OGRS) is a predictor of re-offending based age, gender and criminal history (static risk factors). A score of 1 indicates a 1 percent chance that a service user will re-offend within a year and a score of 100 indicates a 100 percent chance of re-offending within a year. The average (mean) OGRS score for the 27 service users on the pilot was 50.37 ranging from 5 to 94 . This is a slightly higher average than for the general caseload across the three CRCs. During interviews many service users described their current lives and their lives leading up to their offence. Common issues mentioned by service users were: being homeless; relationship breakdowns; loss of contact with families; influence of delinquent peers; unemployment; substance abuse; bereavement; isolation; mental health issues; and anger management. These selfassessments were consistent with analysis of the Offender Assessment System (OASys). Analysis of the service user's OASys assessments found that the proportion of service users in the pilots with a need was consistently higher compared to the general population of service users in the three CRCs. Rates of need were particularly high in relation to alcohol; drugs; attitudes; emotional wellbeing; lifestyles; relationships; and thinking and behaviour.

To assess service user well-being an established psychometric measure (Short Warwick-Edinburgh Mental Well-being Scale, University of Warwick, 2015) was administered to service users at two. Of the 27 who participated, 18 completed the questionnaire pre intervention and 12 post intervention. Seven completed both the pre intervention and post intervention questionnaires. Mean scores for all responses (including matched and unmatched pairs) were 20.53 (S.D. 3.31) pre-intervention and 22.35 (S.D. 4.33), postintervention. Mean scores for matched pairs ( $N=7$ ) were 21.47 (S.D. 3.21) pre-intervention and 22.35 (S.D. 5.52) post-intervention. These are lower than mean scores for general population surveys in England. However, it would be unwise to draw conclusions about the quantified difference between well-being for this group of service users compared to the general population, given the very small numbers involved. 
We assumed that sufficient levels of self-efficacy in both staff and service user groups would be required for personalised working, particularly 'deeper' models of personalisation such as co-production. To test the feasibility of measuring self-efficacy we administerd the Generalised Self-Efficacy scale (Schwarzer \& Jerusalem $1995)$ to staff, volunteers and service users before and after their engagement in the personalisation project. The mean staff pre-intervention score was 32.00 (S.D. 2.45), and the post-intervention score was 33.29 (S.D. 3.20). The mean service user pre-intervention score was 27.39 (S.D. 4.24), with a post-intervention score of 27.58 (S.D. 4.25). Due to very small sample sizes it is not possible to draw conclusions about the results.

\section{Findings}

\section{Training}

Generally, staff found the training programme useful and reported that it improved their knowledge of person-centred practice. Staff appreciated the opportunity to speak with experts from the social care sector and learn new skills. Some staff felt that the training did not offer sufficient advice on how to combine the person-centred practice tools with the need to manage risk. For example, practitioners felt that some of the tools and questions may highlight additional risk factors and that it would not be appropriate to use them with certain types of service users.

"The training was done by a social care expert, her background failed to account for criminal justice and that some of the concerns held by those who attended the training were not recognised and skimmed over." (staff)

\section{Person-centred practice tools}

Some qualitative data from the evaluation suggest that the tools have supported greater understanding and connection between practitioners and service users and suggest that the tools can facilitate co-production between staff and service users. Although staff suggested that they already worked in a personalised way, some mentioned that the tools allowed for them to acquire more knowledge of the service users they worked with, beyond that of which usual practice allowed for. Some staff felt that using the tools increased engagement with service users. Staff explained that the tools improved communication, increased rapport and encouraged a positive relationship, a relationship which focused on a service user's assets. In a number of cases they have enabled more information to be gathered to support the creation of positive tailored support plan.

Most practitioners reported that the tools increased their face-to-face contact with service users, particularly early in an order and led to them taking a more intensive and interactive approach to their case management sessions. However, other practitioners failed to make full use of the tools. One reason for this was a lack of meaningful time spent with service users due to completing tasks detailed in the sentence plan, issues with engagement and high workloads. At least one member of staff felt that the demands of the organisation and requirements of personalisation were contradictory. Some staff felt that the use of the tools was not appropriate in the initial stages of a supervisory relationship and staff also felt tools were not appropriate when clients were in crisis.

I think using the tools during crisis point is unfeasible in our current setting. Women's lifestyles can be very chaotic and extreme crisis point so practical help is the core concern when a service user enters the centre. (staff) 
It is noteworthy that staff tended to see the challenge in implementing the tools in terms of changing their 'questioning style', suggesting that more 'radical' elements of personalisation such as co-production had yet to be fully embedded. Similarly, some of the qualitative data suggests that while staff appreciated the tools and found them valuable, their main value was to increase staff confidence in their risk assessments rather than because they led to greater co-production. Some staff commented on feeling in control when using the tools and that using tools that allowed them to gather more and better quality information about service users, particularly the risks they presented, and this made staff feel empowered.

I felt some of the tools were great in gathering the information from service users. I would definitely like to use the tools in the future. It helps opening up service users and gathering as much information as possible. I especially liked the tools because you did it on yourself. I thought this was really good. (staff)

Some service users mentioned that using the tools made them think differently about their lives, recognise how their behaviour affected both themselves and others around them and better understand some of the factors that influenced their thinking and actions. Some evidence showed that tools encouraged service users to take responsibility by helping them to develop a sense of direction. Overall, service users enjoyed using the tools. Several mentioned finding the one page profile tool especially beneficial as it helped them to get things down on paper and provoked discussions that made them think differently about certain aspects of their life allowing them to keep track of this and reflect on the progress they had made.

We did a pros and cons exercise and that made me think about getting into a routine. Just knowing where I am and what I need to do just helps me deal with the problems of the day. (service user)

I think that I have learnt more about me. I can see that there are different ways of doing things than how I was getting on. I think the way that she asked the questions, it really made me think differently; like the good day/bad day sheet that we did. (service user)

\section{Sentence plans}

Practitioners reported that person-centred practice allowed them to explore a service user's life as a whole thus making the sentence plan more holistic and better able to take into account issues which service users considered to be important such as their families and friends. Practitioners reported that employing personcentred practice allowed them to get to know more about the service users earlier in the Order and this meant that they were better positioned to create a plan specific to their needs. Service users mentioned that the sentence plan incorporated areas of their life that extended beyond the issues typically included such as housing, drugs and alcohol and relationship support to include factors that would enable rehabilitation. For some service users the sentence plan facilitated better personal organisation in their life and offered a sense of direction and structure.

Having a more engaged and transparent sentence planning process and a sentence plan over which clients felt more ownership had, in some cases, helped them re-appraise their lives and set new goals. In some cases the plan appeared to offer a mechanism of tracking/reflecting on progress achieved so far whilst also remaining focussed on future aspirations. Some service users explained that this process did stimulate thought and was useful in mapping out the steps required for them to achieve and surpass some of their rehabilitative goals.

I did like doing the plan together as I think that this helped me focus on what it was I needed to change."(service user) 
Most of the service users interviewed reflected on the collaborative process of sentence planning and their personal ownership of the plan. Practitioners and service users talked about the planning being collaborative and generated through conversations which helped to identify areas of support needed and encourage service users to speak about elements of their own life. Service users mentioned their Case Manager explaining to them that the plan was theirs to complete and it was up to them to decide what they wanted to include in it, and that the Case Manager would provide support should they require it.

"Each week we would do something different; I know that there is all sorts of help available. I remember her saying "It's your plan" and we used that as a guide about the stuff that I wanted to do. It didn't miss anything out; everything that I wanted was in there." (service users)

Service users appeared to respond well to this and several service users were able to describe their planned approach to their rehabilitation and highlighted areas to which they felt required immediate action.

While, generally sentence planning seemed to reflect a move towards co-production, this wasn't always the case. A common issue was that service users and their Case Managers did not feel able to explore wider and more personal goals whilst they had pressing and urgent basic needs such as accommodation, or engaging with drug treatment.

I can't really think when I'm thinking about sleeping outside. (service user)

The only goal I have at the moment is to get housing, my case worker knows this. (service user)

Some service users could not remember completing a plan, some of the reasons for this included them having more immediate concerns such as appearances in court or hospital visits.

Done the plan but can't remember the detail of it. I can remember setting goals and identifying what people I would need to achieve goals (service user)

\section{Case management}

Depending on the case, each service user was required to attend either weekly, fortnightly or monthly supervision meetings. According to circumstances these took place both in the CRC office and the community. Most service users reported sessions lasting between 30 minutes and an hour. Both service users and staff described their relationship in several instances as being one that was positive, forward thinking and goal orientated. Almost all staff mentioned that they delivered practice in a way that was open and friendly whilst putting an emphasis on development of the relationship as being hugely important in making meaningful changes to a service user's life.

Service users talked about receiving advice and guidance from Case Managers and being able discuss some of the priorities in their life in an open and honest manner. This allowed practitioners to develop a connection with the client and reassured service users that help was there should they require it. Practical support was important to both service users and Case Managers. Some service users said that they wanted help with substance issues, relationship issues, mental health support and accommodation. Generally they felt supported and that their Case Manager was able to help them access services. Some service users felt that a more personalised way of working had allowed service users and staff to think in greater depth about their problems and see things from a different perspective, resulting in more useful practical support being offered: 
Absolutely she can help me... She has chased stuff up for me; made some calls and looked into things for me; this didn't happen before. (service user)

Besides practical support, service users described that Case Managers motivated them and constantly reminded them of their goals to ensure they remained focussed. Case Managers gave service users belief and positive encouragement. Case Managers helped service users to remain positive and optimistic even when they faced difficulties and service users described Case Managers making an emotional investment in their rehabilitation.

Some service users described a period of 'getting to know one another' and that this was an important stage in developing trust. Both staff and service users discussed the importance of having an open and trusting relationship. Case Managers described how relationships that were less structured or prescribed and more responsive to need could result in a more personal and relaxed relationship that was nevertheless a professional relationship. Practitioners often referred to their practice as being professional with clear boundaries stressing the importance of being clear and consistent while treating those they work with equally. One service user described their relationship with their Case Manager as "a very healthy and professional relationship" (service user), others talked about their Case Manager being a friend, but often emphasised the professional nature of the relationship as well.

I don't feel like a criminal. I've been for a coffee and a chat with her; not necessarily making friends just someone I can confide in. I think that there is a lot of trust in the relationship and its nice to know that someone believes in me. (service user)

It was evident that service users and Case Managers were able to discuss goals and plan ways activities that would help them to achieve these.

She is honest and lets me know what I need to do...gives me a little nudge sometimes... you know, a nudge in the right direction letting me know that I can do it. She's really helped me. (service user)

Some staff talked about making joint decisions with service users:

I see my role as a guiding role so working with the service user to make joint decisions rather than imposing things on them. (staff)

A number of service users explained that it was up to them to change and that their Case Manager could only do so much. In these instances service users displayed a sense of agency as they took responsibility for their own rehabilitation.

I can't come in here and say to my Case Manager is this is what I want, you do it, obviously that is not what she is there for. Obviously I need to be willing to take the steps for myself. (service user)

In a number of cases service users spoke about feeling understood and listened to. A common theme was that service users appreciated being listened to and because of this it made them feel valued and cared for. When service users felt listened to, they found it easier to talk and hold meaningful conversations (something they often find difficult with others) which allowed for both parties to get to know one another better. Some of the service users described how their Case Manager understood their priorities due to their Case Manager creating the space to listen and allowing open conversations:

She is always herself; caring and supporting and I feel that we can sit down and talk. She gives me the right advice to see the right people. She is interested in priorities. (service user) 
An important element of the person-centred practice pilots was to concentrate more on a service user's strengths or assets. It was observed that Case Managers gave mostly positive feedback and while they could have focused on more negative aspects of a service user's life, they instead choose to highlight instances during which progress was evident whilst assuring their practice was asset/strengths based.

I like the fact that because of this approach we can be less about the negative and imposing controls and be more focussed on the service users strengths. This feels like a more positive focus, I feel like I am able to use desistance theory more in my work. (staff)

Many staff focussed on the positive aspects of the service user's life whilst looking to build on change. Some service users talked about feeling positive about themselves and felt empowered due to their Case Manager believing in them. Others felt encouraged and generally regarded seeing their Case Manager as a positive experience.

She just wants me to do well and give me all the right positive vibes. (service user)

I leave these appointments feeling really positive and believe in myself. (service user)

Almost all service users understood their role in the probation process as complying with their order, doing what was asked of them, attending on time and engaging with services. They often mentioned that it was their responsibility to listen to what their Case Manager said and comply with what is asked of them whilst better understanding themselves and the reasons for their actions. They also recognised the statutory elements of their order and saw it as something they need to address to allow them to move on with their life.

I'm taking what I can from it and learning from it. I know that I need to be on time etc. and take things that she is telling me onboard and try and understand the reasons why I did what I did. (service user)

\section{Enabling fund}

Sixteen service users across Pilot Sites 2 and 3 were eligible to access enabling funds. By the time data collection for the evaluation had finished only 4 had done so. In one instance a service user spent the majority of the funding allocated to them by enrolling on a 15-hour intensive driving course (one theory test and practical test) at a cost of $£ 370$, the same service user made use of the remaining sum by purchasing an annual gym pass at a cost of $£ 181$ making a total expenditure of $£ 551$. The gym membership was purchased to allow the service user to improve his own mental and physical well-being. One service user used $£ 82.85$ to purchase a passport to give them a form of identification to replace their driving license which they lost after being banned from driving. The passport allowed for them to set up a bank account and apply for work. One service user spent $£ 517.90$ on rent arrears to allow them to move up the housing list and increase their likelihood of attaining appropriate housing in the future. Another service user spent $£ 134.34$ on a bike to allow them to travel to work more easily.

None of the service users spent the total amount allocated to them and none of them pooled their funds. At the point data collection for the evaluation was completed some of the service users were in the early stages of identifying spending options linked to their sentence plans. In some cases service users spoke about waiting for the right moment or anticipated a future situation where spending might be needed. Some service users were already in stable jobs and accommodation and felt less inclined to access the fund due to already having 
the sufficient resources to support themselves. Other service users simply reported that they had not yet got around to spending the fund. In some cases goods or services identified by service users were actually available through other routes. Service users who had not accessed funding at the point of writing considered using the funding for purchasing furniture, purchasing a special mattress to improve poor sleep patterns, paying deposits for accommodation, a heavy goods vehicle licence and a training course to qualify as an electrician.

Practitioners mentioned their initial excitement of having access to the funding but then went on to state that it had taken service users much longer than expected to use the fund. Some practitioners felt that they needed to have worked with the service user for a significant amount of time and engaged with them whilst they were stable in order to identify and spend the money on something meaningful.

When the women first come out of prison its not always completely obvious what exactly they should spend their money on in order to make lasting change to their lives. (staff)

Setting up processes by which enabling funds could be spent was complicated. The two sites using the enabling fund established different proceses but both involved several authorisations and some documentation which slowed down access to the fund. Spending the fund also raised issues of client confidentiality with concerns in at least one case that if the CRC paid for a service, this would reveal that the service user was an offender.

Service users reported that knowing they had access to the enabling fund meant they were able to exercise choice over the goals they wanted to reach and that should any barriers present themselves service users would have more control in overcoming them.

I've not used the fund but that's not to discredit it. Knowing it's there gives a security...l've got it if it's needed. My main goal was college. I've not needed it yet but it gave me the extra push knowing I could use the fund if I needed it. (service user)

Service users talked about the enabling fund increasing their trust in their Case Manager and that the funding allowed them to think differently and consider other areas of support. Knowledge of the funding also had a positive effect on Case Managers. One reported that knowing they had access to and enabling fund allowed them to be flexible with service users and that it made them feel more confident in their ability to help as previously funding often presented a barrier, now they had the means to overcome this.

Having access to an enabling fund made me feel more confident in helping other services users, this is because it would be really appropriate should I encounter any barriers or start to get frustrated because progress is not being made.... The service users who did spend the money were overwhelmed at the support they had, especially [the service user] who spent it on rent arrears, this changed her life. (staff)

However, not all service users that were interviewed had a good understanding of the enabling fund. Some service users seemed to know they had money available to them but little else. Some were sceptical that the funding really existed. When questioned about the enabling fund some service users failed to recognise it all. Some Case Managers explained that service users had forgotten it existed.

To test the effect of knowing the amount of the enabling fund has on the choices that service users make, we asked Case Managers to inform some service users how much funding was available for them and in other cases to inform them of the enabling fund but not specify a maximum amount that could be spent. Just one service user was eventually told how much of the enabling fund they were entitled to and this individual was one of those who accessed funding. Those who knew about the fund but not about the amount available reported being cautious about spending the money because they wanted it to "mean something" and were waiting for the right opportunity. In one case a service user explained that not knowing of the amount 
deterred them from spending it and encouraged them to think long and hard about what they wanted to achieve and how the fund could help them in the long run:

I don't know how I could use or how much over how long a period. I wanted to fund the really mean something so I have not used it so far. (service user)

While reception of the enabling fund by staff and clients has been broadly positive, the evidence gathered does not demonstrate that co-production has been central to decisions on how to use the funding. When personal budgets were introduced in social care there were concerns that they would be 'misused'. The same was true in this pilot. Some staff were concerned that the enabling fund could have the potential to be misused and required an increased amount of monitoring to ensure it was being used correctly. Some staff spoke about the enabling fund having the potential to be used for purposes other than rehabilitation and that some service users would not be suitable. Some Case Managers initially withheld knowledge of the funding on the basis that the Case Manager should only disclose the funding to service users once they had identified a need for goods or services. Others used their discretion to decide which service users to tell about the fund. Such concerns and strategies suggest that while the presence of the funding might have encouraged some elements of personalisation, co-production was not yet embedded within the pilot.

\section{Service user attitudes to personalisation}

Service users who had previously had contact with probation services were asked to compare their experience on the personalisation pilot with their previous experience of probation. These results report the experiences of a small number of service users and so must be treated with caution.

When service users remembered being on probation in the past and compared it to their current experience on the personalisation pilot, many of them recognised a different approach and were more positive about their current experience. Some suggested that during previous orders probation seemed more punitive and strict. For instance, one service mentioned that probation seemed to be more relaxed these days and they were treated with more respect. One service described being given more one-to-one time with their Case Manager. Some service users spoke about having a better rapport this time than on previous orders and that probation had a more caring and supportive feel to it:

Probation has felt really different this time. I know that its mandatory but it always felt like all punishment and no support but this has been different. In a weird way it has also been enjoyable and a lot more welcoming. Before it used to feel all about the sanctions and I used to be really afraid of being late. Now I come in and get a coffee and its much nicer. (service user)

Probation is more personalised than before. The start of the licence was more rigorous and intrusive this time but I can now see the benefits. I would say to a new service user to do what you can, you couldn't have picked a better time. Before [when on previous orders/licence] nothing was available you couldn't get on a course, they didn't have partner agencies. This time because of entitlement [to funding] there is more in play if you want to change. Having access to some money has made me feel like moving forward is possible and that I can finally do what I want with my life. (service user)

Two provisos should be made. First, some of the positive changes noted by service users were probably due to the new 'Interchange' approach being implemented across the CRCs that included a more personalised service, new offices and additional partnership working. Secondly, the positive view of probation compared to previous experiences was not universal. Some of the service users who previously been on probation could not 
recall whether their experience was positive or negative. Some had last been on probation up to ten years ago meaning they were unable to remember the details of their order. One service user felt that probation was better in the past and that this time it was too intrusive.

\section{Organisational context}

The pilot took place at a time of considerable organisational change. Staff were still getting used to the transition from Probation Trusts to the newly formed Community Rehabilitation Companies. Those we interviewed consistently mentioned these changes. Many staff had experienced changes to their role and title and in some $\mathrm{CRCs}$ a redundancy programme had contributed to uncertainty among staff. Staff regularly mentioned that new IT systems did not always work as intended and that this hampered their work. Office moves were also often disruptive with many staff going through office refurbishments or moves to new office locations during the pilot.

Some staff were concerned that they were struggling with a high workload and that this posed considerable challenges to implementing the pilot. Staff felt that the personalisation work required more time than the standard approach to case work, particularly in the early stages of an order.

Staff need to be allowed to work differently and that current workloads do not allow for this. For personalisation to work practitioners need to be given the space to conduct meaningful work. (staff)

Practitioners explained that morale in the office was often low due to staff being unable to keep up with performance measures and work demands which, at times, seemed unreasonable. This meant that Case Managers on the personalisation project were often unable to work in new ways and increasingly likely to resort to working in their 'comfort zone'. The high workload has also meant that staff were unable to undertake additional learning and training opportunities:

The requirement to keep up with performance management targets was a common concern among staff and many felt that meeting these targets constrained their work on the pilot. Not being able to get work done meant staff sometimes felt demotivated as they struggled to get tasks completed, working outside of work hours to stay on top of their caseload. Most staff recognised that the organisation was going through a period of transition and that many of their concerns were as much due to high workloads and performance management pressures as they were the personalisation pilot. Staff stressed the importance of effective management, stating that their line managers had been exceptional in keeping them motivated and confident.

However, Case Managers did not generally believe that their line manager could help with workload and performance management issues because this was overseen by more senior managers. Staff talked about the need for a reduced workload to facilitate implementation of the project and some staff reported requesting this, but with limited results.

\section{$\underline{\text { Discussion }}$}

The aim of these pilots was to test the viability of each personalisation element in a 'real world' setting, to established whether the elements were implemented successfully and to learn implementation lessons. Elements to support personalised practice in the criminal justice were developed based on the model in social care. These pilots focused on how PCP could facilitate personal change and support choice and control of rehabilitation. When findings are reviewed against these elements, we conclude that overall, the pilots were implemented as planned, with a broadly positive experiences among staff and service users. 
Some staff involved in the pilots felt they benefitted from person-centred practice having used the various tools because they felt they had a better understanding of service users' lives and were better able to work with service users to set goals that took account of the service users' holistic needs as oppose to their more narrowly conceived criminogenic needs. Staff and service users felt that they were able to develop more productive and trusting relationships that provided safe spaces for service users to discuss issues underlying their offending behavior. Both staff and service users felt that they were able to do this within appropriate professional boundaries. There was a recognition that such relationships took time to develop and that the supervisory relationship was therefore a dynamic one. Most of the service users identified elements of collaboration process in sentence planning and ongoing work with their officer. They often expressed some personal ownership of the plan, and that this had a positive impact on their behaviour and attitudes. Some service users talked about feeling positive about themselves and empowered due to their Case Manager believing in them others felt encouraged and generally regarded seeing their Case Manager as a positive experience. Service users conveyed a sense of having more choice and control.

Personalisation encompasses a range of new ways of designing services, which can provide both what Leadbeater (2004) describes as 'shallow' and 'deep' approaches. These can range from relatively shallow approaches such as 'providing people with a more customer-friendly interface', 'giving users more say in navigating their way through services', and 'giving users more direct say over how money is spent', to deeper approaches such as users being 'co-producers of a service', and self-organisation (Leadbeater 2004 p.21 - 24). Following Leadbetter and thinking about personalisation as covering a range of approaches from shallow to deep, there is certainly evidence from pilots of 'shallow' models of personalisation starting to emerge. Within the evaluation timeframe the implementation of components of a person-centred approach has had some positive impacts on probation supervision and these have been felt by service users. However, the extent of the change and how embedded it is are uncertain. It does seem that person-centred practice freed up staff to raise the standards of their practice, particularly in developing relationships with service users and enhanced the use of professional discretion in their work. Thus, personalisation has provided service users with a more customer-friendly interface and given then more say in navigating their way through services. But, the potential of personalisation has not been consistently understood. At the start of the pilot some staff thought they were already doing person-centred practice, but their behaviour did not always demonstrate this. During the pilot there was some behavior change among staff and service users, but changes were modest and fell short of a 'paradigm shift' in practice. Some staff tended to interpret person-centred practice as about being friendly and relaxed with service users, while service users still looked for direction from their Case Manager in defining their needs, setting priorities and accessing services. While supervision had a more 'individualised' feel it fell short of co-production: enabling service users to exercise significant choice and control over their rehabilitation. Experience in social care suggests that this does take time, both in the length of relationship between staff and a service user, which was not possible in this pilot, and in terms of practice maturity to enable staff to gain confidence and develop their skills in co-production. This pilot reflects early experience in personalisation in social care, and more fundamental change requires a longer-term process. Although a new case management system with elements of personalisation is being rolled out across the five CRCs, during the pilot work person-centred practice was not integrated into current working practices or systems. More thought is required on overall process design.

Drawing on a range of sources Pearson et al. (2014) identify three levels of personalisation: prevention, participation and choice. Findings from the pilots can be usefully considered in light of these three approaches.

\section{Prevention}

Personalisation can be seen as a means of prevention, designed to build an individual's capacity to manage their own lives. In a criminal justice context a key challenge emerging from the pilots was to reconcile building individual capacity with managing criminogenic risk. Throughout the pilot the balance between managing 
criminogenic risk and adopting a more asset-based approach was a challenge for staff. Staff raised concerns that risk is dynamic and the appropriate balance between risk management and promotion of service user assets might change, sometimes rapidly, necessitating a recalibration of staff/service user relationships. During training some staff questioned whether the design of personalisation tools was based on an adequate understanding and appreciation of the need to manage criminogenic risk and during implementation some staff raised concerns about the balance between risk management and asset-based working.

For Ward and Maruna (2007), the solution to reconciling this seeming tension between 'risk' and 'agency' is that in asset-based, co-produced approaches to rehabilitation where people are conceptualised to be part of complex systems, risk is viewed as multi-faceted rather than purely individualistic. A critical task is thus to manage the balance between promoting the 'good life' and reducing risk:

Simply seeking to increase the well-being of a prisoner or a probationer without regard for his or her level of risk may result in a happy but dangerous individual. Alternatively, attempting to manage an individual's risk without concern for goods promotion or well-being could lead to punitive practices and a defiant disengaged client. (Ward and Maruna 2007 p.125)

This suggests that in the early stages of a supervisory relationship there is a greater focus on managing risk, but promoting agency and a 'good life' are nevertheless a concern, and as the sentence progresses there is a greater focus on promoting agency (but managing risk nevertheless remains a factor). Of course, in reality, risk is dynamic and so a sentence is unlikely to progress in such a measured and linear fashion. There are suggestions within the data of this approach starting to emerge. Some staff described how the use of personalised approaches allowed them to better understand a service user's criminogenic risk, for instance, by being better able to place specific incidents within the context of the service user's whole life. Within the scope of this smallscale pilot it is not possible to say conclusively whether this better appreciation of risk was due to a more personalised approach or simply that staff invested more time into relationship building early in the sentence. Also, the pilots were of short duration and so such approaches could not fully develop within the timescales.

\section{Participation}

Personalisation and particularly 'co-production' as an element of personalisation is a means of allowing people with complex needs to participate in shaping and delivering their service solutions. Drawing on earlier works, Pearson et al. (2014 p.18) suggest that "co-production describes a particular approach to partnership between people who rely on services and the people and agencies providing these services." This raises important questions in a criminal justice context where interactions between clients and agencies are mandated.

Participation also has a social dimension. For Pearson and colleagues:

Over and above the focus on enhanced individual support, classic co-production relates to the generation of social capital - the reciprocal relationships that build trust, peer support and social activism with communities. (Pearson et al. 2014 p.18)

Recent thinking work on desistance has sought to bridge explanations of desistance that err towards structure and those that err towards agency. For example, Weaver (2012) advances a relational explanation of desistance in which 'reflexivity' and 'reciprocity' are key concepts to understanding how desistance is coproduced. Emphasising the relational context within which desistance is co-produced leads Weaver to argue that correctional interventions must progress beyond a focus on the individual to a focus on generating social capital and that social capital exists within social networks shared by people in reciprocal relations. Therefore, 
Weaver (2012 p.12) envisages criminal justice interventions that "focus less on the structural outcomes derived from participation in a given network and more on the connection between people and on fostering the reflexivity of the individual who is being connected by the good of the relationship".

In some cases the approach adopted by Case Managers was encouraging service users to build capacity to manage their own lives. We also found some examples of 'co-production' particularly around sentence planning. However, while relations between Case Managers and service users showed elements of trust and reciprocity, it was not obvious that this extended to relationship building between Service users and their families and communities. Building social capital did not seem to be a key element of work undertaken by Case Managers and Service users.

\section{Choice}

Personalisation is sometimes also portrayed as a means of embedding consumer choice linked to a broader discourse which emphasises the potential of personalisation to transform relationships between the state, service providers and service users (Pearson et al. 2014).

One mechanism to promote choice explored in the pilots was the Enabling Fund. The Enabling Fund was only just starting to be used when the evaluation reported and so early findings must be treated with caution. In the social care sector when personal budgets were introduced, many were concerned about the abuse of such budgets, but these concerns were not realized (Fox 2012). The limited use of the enabling fund in this project and in other projects where a similar enabling fund has been used (eg Clarke, Fox and Sefton 2014) suggests that both staff and organizational concerns about the misuse of funding are likely to be misplaced.

We found little evidence that personalisation was embedding consumer choice or noticeably increasing service user control over the services they received. While the presence of the enabling fund has led to increased feelings of choice and control among some service users, in reality staff have largely retained choice and control and in some cases have made a conscious decision not to implement the fund. Perhaps the limited elements of personalisation demonstrated during the pilot are not surprising. It takes time to build a trusting relationship and co-produce services and the pilot timescale was not long enough for the approach to be fully realised during the evaluation period. Service users did not always feel able to explore wider and more personal goals whilst they had pressing and urgent basic needs such as accommodation, or engaging with drug treatment. There are echoes here of the experience in social care with rolling out personal budgets. As Fox et al. (2013) describe, in the social care sector some local authorities have appeared reluctant to cede genuine budgetary control to individuals, with some local systems involving a high degree of professional input into allocating resources and choosing services.

\section{Conclusion}

A model of personalisation in criminal justice was developed, based on the model in social care. The pilots described in this paper explored how person-centred practice could facilitate personal change and support choice and control of rehabilitation. When findings are reviewed against these elements of the model, it is reasonable to conclude that overall, the pilots were implemented as planned, with positive experiences for many staff and service users.

Tools to support personalised working and training for staff to help them make use of the tools were designed and delivered. In all sites service users were recruited and experienced the different personalisation concepts. Broadly, staff appeared to be enthusiastic, positive and welcoming in their attitude to changes in practice, though some staff on one site were sometimes negative in their approach to the proposed changes and this made it difficult for the pilot to maintain its momentum. Many service users noticed differences in the service 
they received and reported a more positive experience of probation supervision compared to previous sentences.

However, implementation was not without challenges. Across the three sites caseloads were high and new performance management processes implemented by Interserve across all five of its CRCs were experienced by staff on the pilot as onerous. Generally, workload relief for staff on the pilot did not happen as planned. It was challenging to achieve target numbers of service users within project timescales and more service users disengaged from the process than expected. Some staff were uncomfortable with some elements of the personalisation concepts being piloted and did not fully implement them. For example, at least one member did not fully implement the enabling fund and there were reservations about how person-centred practice will work alongside dynamic risk assessment and management.

Staff found person-centred practice more time consuming than usual practice, particularly in the early stages of supervision. Although it is hard to quantify, a more person-centred approach also placed additional emotional demands on staff as stronger, more trusting relationships between staff and service users sometimes increased service users expectations about the level of emotional support that their Case Manager should provide alongside more practical support. The CRCs, while recognizing the value of the pilots, struggled to provide staff with workload relief to address additional demands on their time. While managers and fellow staff provided important support to staff involved in the pilot they did this in spite of heavy workloads and competing priorities, raising questions about how sustainable a large scale or longer pilot might be.

Nevertheless, there seems to be sufficient positive experience in this pilot to merit moving to the next stage of the personalisation project, which will develop an integrated model of personalisation, building on the experience of all five pilots ${ }^{1}$. This model will be tested out in a single site over a period of 12 months starting in early 2018. Based on the learning from these pilots it seems that a large pilot of person-centred practice should include many of the elements tested here, but there is a need to further refine tools and training to incorporate risk management more effectively; that staff will need greater support, including a model that is more embedded into mainstream practice; and that further work will be required to promote genuine coproduction between staff and service users.

More broadly, there is some evidence here to support the idea that personalisation has a place in wider public service reform beyond social care. It is not sufficient to simply transfer social care experience to other sectors, but key principles and some tools and practice have the potential to be applied in different contexts and settings.

\section{$\underline{\text { References }}$}

Andrews, D.A. \& Bonta, J. (2006) The Psychology of Criminal Conduct. Newark, NJ: Lexis/Nexis

Clarke, R., Fox, C. and Sefton, M. (2014) London Probation Trust Mentoring Evaluation, Manchester: MMU

Connell JP and Kubisch AC (1998) 'Applying a theory of change approach to the evaluation of comprehensive community initiatives: progress, prospects, and problems', in Fulbright-Anderson K, Kubisch AC and Connell JP (eds) New Approaches to Evaluating Community Initiatives: Theory, Measurement, and Analysis. Washington, DC: The Aspen Institute.

\footnotetext{
${ }^{1}$ The other two pilots concentrated on the 'supply-side' of the 'market' for personalised services and explored approaches to building social capital and network development.
} 
Department for Communities and Local Government (2014) Qualitative Evaluation of the London Homelessness Social Impact Bond: First Interim Report, London, DCLG

Education Endowment Foundaton (2015) EEF evaluation: A cumulative approach. London: EEF

Glasby, J., Le Grand, J. and Duffy, S. (2009) 'A healthy choice? Direct payments and healthcare in the English NHS,' Policy \& Politics, Vol.37(4) pp. 481-497

Fox, A. (2012). Personalisation: Lessons from Social Care, London: RSA.

Fox, C. \& Grimm, R. (2015). 'The role of social innovation in criminal justice reform and the risk posed by proposed reforms in England and Wales', Criminology and Criminal Justice 15(1) 63-82

Fox, C., Grimm, R. and Caldeira, R. (2016) An Introduction to Evaluation, London: Sage

Fox, A., Fox, C. and Marsh, C. (2013). 'Could personalisation reduce re-offending? Reflections on potential lessons from British social care reform for the British criminal justice system,' Journal of Social Policy, 42 (4), $721-74$

Fox, C. and Marsh, C (2016) Operationalising Desistance through Personalisation' European Probation Journal, $8(3), 185-206$

Leadbeater, C. (2004) Personalisation through Participation; A New Script for Public Services. London: Demos.

Maruna, S. (2001) Making Good. Washington, DC: American Psychological Association Books

McNeill, F. (2009) Towards Effective Practice in Offender Supervision, Glasgow: The Scottish Centre for Crime and Justice Research

McNeill, F., Farrall, S., Lightowler, C. and Maruna, S. (2012) How and why people stop offending: discovering desistance, Glasgow: IRISS

McNeill, F. and Weaver, B. (2010) Changing Lives? Desistance Research and Offender Management, Glasgow: The Scottish Centre for Crime and Justice Research

Mackenzie M. and A. Blamey (2005) 'The practice and the theory: Lessons from the application of a theories of change approach' Evaluation 11: 151-168

Ministry of Justice (2013) Transforming Rehabilitation: A revolution in the way we manage offenders, London: Ministry of Justice

Ministry of Justice (2017) Prison Population Figures: 2017 Population bulletin: weekly 25 August 2017 available at: https://PILOT SITE 2.gov.uk/government/statistics/prison-population-figures-2017 (accessed on 25 August 2017) 
Mulgan, G., Tucker, S., Ali, R. and Sanders, B. (2007) Social Innovation: What it is, why it matters and how it can be accelerated. Said Business School, Oxford.

National Audit Office (2016) Personalised commissioning in adult social care, London: NAO

Pearson, C., Ridley, J., and Hunter, S. (2014) Self-Directed Support: Personalisation, Choice and Control. Series:

Policy and practice in health and social care, 19. Dunedin Academic Press: Edinburgh

Prime Minister's Strategy Unit (2007) Building on Progress: Public Services, London: Cabinet Office

Rose, T. (2016) The End of Average: How We Succeed in a World that Values Sameness, New York: HarperOne

Rossi P, Lipsey M and Freeman H (2004) Evaluation: A Systematic Approach: London: Sage.

Roulstone, A. and Morgan, PILOT SITE 2. (2009) ‘Neo-Liberal Individualism or Self-.Directed Support: Are We All Speaking the Same Language on Modernising Adult Social. Care?' Social Policy and Society, Vol.8(3). p.333

Schaufeli, PILOT SITE 2., \& Bakker, A. (2004). Utrecht Work Engagement Scale: Preliminary Manual. Retreived from http://PILOT SITE

2.wilmarschaufeli.nl/publications/Schaufeli/Test\%20Manuals/Test_manual_UWES_English.pdf on 26 October 2017

Scheirer MA (1994) Designing and using process evaluation. In: Wholey JS, Hatry HP, and Newcomer KE. (eds) Handbook of Practical Program Evaluation. San Francisco: Jossey-Bass Publishers.

Schwarzer, R., \& Jerusalem, M. (1995). Generalized Self-Efficacy scale. In J. Weinman, S. Wright, \& M. Johnston, Measures in health psychology: A user's portfolio. Causal and control beliefs (pp. 35-37). Windsor, UK: NFER-NELSON.

Slay, J. (2011) Personal Budgets and Beyond: What Co-production Can Offer Personalisation, London: NESTA Smith L, Bratini L, Chambers DA, Jensen RV and Romero L (2010) 'Between idealism and reality: Meeting the challenges of participatory action research', Action Research 8(4) pp. 407-425.

The University of Warwick (2015). Warwick-Edinburgh Mental Well-being Scale: User Guide Version

2. Retrieved from

https://www2.warwick.ac.uk/fac/med/research/platform/wemwbs/researchers/userguide/wemwbs_user_gui de_jp_02.02.16.pdf on 26 October 2017.

Tucker, S. and Cadora, E. (2003) Justice reinvestment: To invest in public safety by allocating justice dollars to refinance education, housing, healthcare and jobs [Monograph] Ideas for an Open Society, 3

Ward, T. and Maruna, S. (2007) Rehabilitation: Beyond the Risk Paradigm. London. Routledge 
Weaver, B. (2011) Co-producing community justice: the transformative potential of personalisation for penal sanctions. British Journal of Social Work. 41(6) pp.1038-1057.

Weaver, B. (2012) 'The Relational Context of Desistance: Some Implications and Opportunities for Social Policy', Social Policy and Administration, Vol.46(4) pp395-412

Weiss C (1995) 'Nothing as practical as good theory: exploring theory-based evaluation for comprehensive community initiatives for children and families', in Connell JP, Kubisch AC, Schorr LB and Weiss CH (eds) New Approaches to Evaluating Community Initiatives: Concepts, Methods and Contexts. Washington, DC: Aspen Institute 